Bundesgesundheitsbl 2020 - 63:708-714 https://doi.org/10.1007/s00103-020-03139-2 Online publiziert: 21. April 2020

(c) Der/die Autor(en) 2020

\section{Claudia Lampert}

Leibniz-Institut für Medienforschung, Hans-Bredow-Institut (HBI), Hamburg, Deutschland

\title{
Ungenutztes Potenzial - Gesundheits-Apps für Kinder und Jugendliche
}

Gesundheits-Apps erfahren zunehmend Aufmerksamkeit und aktuell - u.a. durch das am 08.11.2019 im Bundestag beschlossene Digitale Versorgungsgesetz (DVG) - verstärkt Rückenwind aus der Politik. Aufgrund ihrer vielfältigen technischen Möglichkeiten scheinen sie auf den ersten Blick für Prävention, Gesundheitsförderung, Behandlung und Selbstmanagement gleichermaßen attraktiv. Entsprechend umfangreich, breit gefächert und von unterschiedlicher Qualität ist das Angebot an Gesundheits-Apps. Einige Apps erscheinen nur kurz an der Oberfläche, bevor sie von anderen Angeboten wieder verdrängt werden. Nur wenige Gesundheits-Apps setzen sich längerfristig auf dem Markt durch und werden auch von den Nutzerinnen und Nutzern über einen längeren Zeitraum verwendet.

Der vorliegende Beitrag nimmt mit den Gesundheits-Apps für Kinder und Jugendliche einen Ausschnitt des AppAngebotes in den Blick und zeigt anhand dessen auf, wie dynamisch und unübersichtlich der Markt derzeit aussieht. Überdies wird anhand vorliegender Studien dargestellt, welchen Stellenwert Gesundheits- und insbesondere Präventions-Apps gegenwärtig im Medien- bzw. App-Repertoire von Heranwachsenden einnehmen und was bei der Entwicklung von Gesundheits-Apps für diese Zielgruppe(n) bedacht werden sollte. Den Abschluss bilden Handlungsoptionen, die geeignet scheinen, die Potenziale von gesundheitsbezogenen App-Anwendungen besser nutzbar zu machen.

\section{Dynamisch und unübersichtlich - der Markt an Gesundheit-Apps}

Der Markt an App-Anwendungen ist gegenwärtig von einer hohen Dynamik gekennzeichnet, was einen Überblick über das Angebot erschwert [1]. Hinzu kommt, dass Apps in unterschiedlichen Stores angeboten werden, die auf verschiedenen Betriebssystemen (z. B. iOS oder Android) und Geschäftsideen basieren. Zu den großen Anbietern zählen iTunes (Apple), Google Play und Amazon (für Android). Daneben gibt es weitere Stores, die Apps für spezielle Mobilfunkmarken anbieten (z.B. Microsoft Windows Phones, Nokia, Samsung). Bei einigen Smartphones sind GesundheitsApps bereits auf dem Gerät vorinstalliert, die sich mit entsprechenden Wearables (z.B. Apple Watch) verbinden lassen.

Genaue Angaben zur Anzahl an Gesundheits-Apps allgemein oder auch $\mathrm{zu}$ einzelnen Gesundheitsthemen liegen aktuell nicht vor, was u.a. darauf zurückzuführen ist, dass die Kategorie „Gesundheits-Apps“ bisweilen unterschiedlich breit gefasst wird und zudem die Apps in den verschiedenen Stores unterschiedlich kategorisiert werden [2, 3]. Scherenberg und Kramer definieren Gesundheits-Apps als „mobile Anwendungen ..., die zum Ziel haben, das körperliche, seelische und soziale Wohlbefinden positiv und nachhaltig auf Basis wissenschaftlicher Erkenntnisse zu beeinflussen“ [4]. Die Definition von Chan et al. ist etwas breiter formuliert und verzichtet auf eine wissenschaftliche Fundierung als kennzeichnendes Merkmal. „Health Apps“ werden demzufolge definiert als „mobile applications that were aimed towards health promotion, including apps for fitness, diet (nutrition recommendations, calorie counting), behavioral regulation, or carbohydrate counting for diabetes" [5, S. 49]. Gesundheits-Apps werden wiederum unterschieden in Präventions-Apps und Medizin-Apps, die sich nicht nur hinsichtlich ihrer Zielsetzung und Inhalte, sondern - im Fall von Apps, die als Medizinprodukte eingeführt werden sollen - in Bezug auf die Anforderungen und erforderlichen Prüfungen gravierend unterscheiden [6]. Insbesondere Letztere stehen aktuell im Fokus gesundheitspolitischer Diskussionen.

Der vorliegende Beitrag richtet seinen Blick auf die Präventions-Apps und damit auf Angebote, die präventive oder gesundheitsfördernde Zielsetzungen verfolgen, eine breite Nutzerschaft adressieren, derzeit noch keiner (Qualitäts-)Prüfung unterworfen und insofern allen Interessierten über verschiedene Plattformen oder Stores zugänglich sind.

\section{Zum Angebot an Präventions- Apps}

Studien zur Entwicklung dieses Angebotssegments sind ebenfalls rar und beziehen sich oftmals nur auf einen Ausschnitt des verfügbaren Angebots (z. B. Apps zur Förderung der psychischen Gesundheit; $[7,8])$. In einer systematischen Literaturauswertung für den Zeitraum 2008 bis 2016 fanden sich lediglich 24 Studien, in denen 15 Apps zur Förderung der psychischen Gesundheit berücksichtigt wurden [7]. 
Herausforderungen bei der quantitativen Erfassung und Untersuchung des App-Angebots bestehen vor allem hinsichtlich der Eingrenzung des Themenfeldes, der Auswahl der App-Stores und der Ein- und Ausschlusskriterien [2, 9]. Allein aufgrund unterschiedlicher AppProgrammierungen bzw. -betriebssysteme sowie unterschiedlicher kategorialer Zuordnungen in den jeweiligen Stores werden selbst bei Verwendung gleicher Suchbegriffe verschiedene Angebote angezeigt [2]. Überdies unterscheiden sich die Stores auch dahin gehend, in welcher Weise Treffer angezeigt werden. Während bei iTunes in der Regel eine Liste von Treffern zu den jeweiligen Suchbegriffen angezeigt wird, erhalten die Nutzer*innen im Google-Play-Store bei jeder Suchanfrage eine Liste von ca. 250 (mehr oder weniger passenden) Treffern, unabhängig davon, welche Suchbegriffe eingegeben wurden. Unklar bleibt dabei, in welcher Weise vorhergegangene Suchanfragen oder App-Downloads das Suchergebnis beeinflussen. Eine Studie des IMS Instituts for Health Care (IMS; USA) ermittelte beispielsweise im Google-PlayStore und bei iTunes im September 2015 insgesamt 160.000 englischsprachige Gesundheits-Apps [10]. Die Initiative Präventionspartner und Kramer schätzten die Gesamtzahl an Apps im Google-PlayStore im Januar 2017 weltweit auf ca. drei Millionen, darunter ca. $131.000 \mathrm{Ge}$ sundheits-Apps, ca. 13.000 deutschsprachige und etwa 1300 Gesundheits-Apps mit mehr als 50.000 Downloads ([11]; Daten gerundet). Eine eigene kombinierte Stichwortabfrage zu gesundheitsbezogenen Apps für Kinder im Google-PlayStore und bei iTunes (jeweils 15 Sucheingaben mit dem Wort „Kinder“ in Verbindung mit verschiedenen Gesundheitsbegriffen, wie z. B. Gesundheit, Ernährung, Zähne, Asthma, ADHS) identifizierte für den Zeitraum April bis August 2017 insgesamt 66 (deutschsprachige) Angebote, die anhand des Titels und der AppBeschreibung eine gesundheitsfördernde Absicht vermuten ließen [9]. Inhaltlich umfasste das Angebot Apps zu Themen, wie z. B. Zahnhygiene, Ernährung, Anatomie, Entspannung/Yoga. Das letztgenannte Beispiel einer Bestandsaufnahme von Gesundheits-Apps für Kinder ver- deutlicht, welchen großen Einfluss Suchund Einschlusskriterien auf die Treffer haben. Unter den gegebenen Bedingungen können Angaben zum Umfang des Angebotes an Gesundheits-Apps insofern immer nur Annährungswerte und Momentaufnahmen darstellen.

Die Anzahl an Angeboten gibt jedoch keinerlei Aufschluss über die inhaltliche Ausrichtung und Qualität der Angebote. Neben Gesundheits-Apps im oben definierten Sinne werden in den Stores auch diverse (Spiele-)Apps angeboten, die zwar über das Thema (z.B. Obst, Ernährung) oder den Kontext (z.B. Krankenhaus, Arztpraxis) einen Gesundheitsbezug haben, jedoch keine gesundheitsfördernde Intention erkennen lassen. Viele App-Beschreibungen sind schlecht übersetzt (oftmals automatisch), teilweise falsch und oftmals wenig aussagekräftig (z. B. im Hinblick auf die Zielsetzung, Verantwortung der Inhalte, wissenschaftliche Fundierung, das Thema Datenschutz und -sicherheit, weitere Kosten) und bieten daher keine hinreichende Grundlage für die Beurteilung ihrer gesundheitsbezogenen Qualität oder für eine informierte Auswahl [1, 2, 9].

\section{Gesundheits-Apps im Medienrepertoire von Kindern und Jugendlichen}

Verschiedene Studien zeigen deutlich, dass sich die Onlinenutzung von Heranwachsenden zunehmend auf mobile Endgeräte verlagert, womit auch Apps an Bedeutung gewinnen [12-14]. Gemeinhin wird von der hohen Verbreitung von Smartphones sowie der steigenden Bedeutung und vermehrten Nutzung von Apps auf eine gute Erreichbarkeit über App-Anwendungen und damit auch auf ein hohes gesundheitsförderndes Potenzial von Apps geschlossen. $\mathrm{Zu}$ den Lieblings-Apps der Jugendlichen zählen aktuell jedoch vor allem solche Angebote, die - neben Spielen - ihrem Bedürfnis nach Kommunikation und Unterhaltung am nächsten kommen: WhatsApp (84\%), Instagram (48\%), YouTube (37\%), Snapchat (31\%), Spotify (31\%) und schließlich - mit sinkender Tendenz - Facebook (6\%) [14].
Zur Nutzung und zum Stellenwert von Gesundheits-Apps im Medien- und App-Repertoire von Kindern und Jugendlichen liegen bislang nur wenige Untersuchungen vor. Auch fehlen Studien darüber, zu welchen Themen und nach welchen Kriterien Heranwachsende Gesundheit-Apps (aus-)suchen [5, 15, 16]. Bei jüngeren Kindern kommt erschwerend hinzu, dass häufig die Eltern über die Auswahl der Apps entscheiden. Auch hier fehlen Befunde, nach welchen Kriterien die Eltern ihre Auswahl treffen, welche Rolle präventive oder gesundheitsfördernde Überlegungen dabei spielen und wie Eltern gesundheitsfördernde Angebote finden bzw. davon Kenntnis erlangen. Vorliegende Befunde zur Nutzung von Gesundheits-Apps beziehen sich daher vor allem auf Altersgruppen, die bereits selbstständig darüber entscheiden, welche Anwendungen sie auf ihrem mobilen Endgerät installieren und nutzen.

Laut einer repräsentativen US-amerikanischen Studie unter 13- bis 18Jährigen haben $84 \%$ der Jugendlichen schon einmal online nach Gesundheitsinformationen gesucht, ein Fünftel (21\%) eine Gesundheits-App genutzt, $12 \%$ ein gesundheitsbezogenes Computerspiel gespielt und $7 \%$ ein Wearable (z.B. ein Fitbit-Armband) verwendet [16, 17]. Allerdings stellt die Suche nach Gesundheitsinformationen keine regelmäßige Aktivität dar: $38 \%$ suchten ein paar Mal im Jahr online nach Gesundheitsinformationen, ein Viertel seltener. Der Anteil derjenigen, die mindestens einmal im Monat online nach Gesundheitsthemen suchten, lag ebenfalls bei $25 \%$. Im Vordergrund steht die Förderung der eigenen Gesundheit, weniger die Suche nach Diagnosen, Behandlungsmöglichkeiten oder Informationen für andere. Entsprechend zählen Sport und Fitness sowie Diäten/Ernährung zu den zentralen Themeninteressen der Heranwachsenden, gefolgt von Stress/Angst (19\%), sexuell übertragbaren Krankheiten und pubertätsbezogenen Themen (jeweils $18 \%)$, Depression/psychischen Themen und Schlaf (jeweils 16\%) sowie Alkohol/Drogen, Hygiene und Erkältung/ Grippe (jeweils 12\%). Das Interesse an Sport/Fitness und Ernährung spiegelt 
sich auch in der App-Wahl wider: $23 \%$ der Jugendlichen, die ein Smartphone besitzen, hatten eine Bewegungs-App heruntergeladen, $14 \%$ eine ErnährungsApp oder einen Kalorienzähler.

Für die Wirksamkeit von Gesundheits-Apps ist jedoch entscheidend, dass sie nicht nur heruntergeladen, sondern auch (regelmäßig) genutzt werden. Die Daten der Studie verweisen darauf, dass dies nur bei knapp der Hälfte (45\%) der Fall ist, während $47 \%$ der Befragten angeben, die auf dem Smartphone vorhandene App kaum oder gar nicht $\mathrm{zu}$ nutzen. Lediglich $8 \%$ gaben an, die Gesundheits-App oft $\mathrm{zu}$ verwenden. Das Geschlecht und der Body-MassIndex (BMI) erwiesen sich dahin gehend als signifikante Prädiktoren, dass insbesondere weibliche Jugendliche und Jugendliche mit einem geringeren BMI Gesundheits-Apps regelmäßig nutzen. Ein Viertel der Befragten, die sich eine Gesundheits-App heruntergeladen hatten, war mit dieser sehr zufrieden (57\% etwas zufrieden, $17 \%$ nicht bzw. gar nicht zufrieden). Diejenigen, die zumindest etwas zufrieden mit der GesundheitsApp waren, schätzten insbesondere die Möglichkeiten der Datenaufzeichnung (63\%), die motivierenden (36\%) oder erinnernden Funktionen (34\%), den Informationsgehalt $(27 \%)$ oder den Unterhaltungswert (25\%). Knapp ein Drittel (32\%) aller Befragten gaben an, ihr Gesundheitsverhalten aufgrund von Onlineinformationen verändert $\mathrm{zu}$ haben. $28 \%$ schrieben diese Veränderungen Informationen aus dem Internet zu, 7\% der Nutzung von GesundheitsApps, $2 \%$ gesundheitsbezogenen Spielen und $1 \%$ der Nutzung von Wearables. $64 \%$ derjenigen, die Gesundheits-Apps genutzt haben, stellten nach eigener Auskunft keine Auswirkungen auf ihr Gesundheitsverhalten fest.

In einer weiteren repräsentativen Studie, in der Jugendliche und junge Erwachsene zwischen 14 und 22 Jahren befragt wurden, wurden GesundheitsApps gleich nach Onlineinformationen (87\%) als Informationsquelle für gesundheitsbezogene Themen genannt [18]. Mit $64 \%$ fiel der Anteil derjenigen, die schon einmal eine Gesundheits-App genutzt haben, vergleichsweise hoch aus. Wur-

Bundesgesundheitsbl 2020 $63: 708-714$ https://doi.org/10.1007/s00103-020-03139-2 (c) Der/die Autor(en) 2020

\section{Lampert}

\section{Ungenutztes Potenzial - Gesundheits-Apps für Kinder und Jugendliche}

\section{Zusammenfassung}

Gesundheits-Apps erfahren zunehmend Aufmerksamkeit und verstärkt Rückenwind aus der Politik. Der Markt ist aktuell durch eine sehr hohe Dynamik und Unübersichtlichkeit gekennzeichnet, die es einerseits unmöglich macht, das Angebot an Gesundheits-Apps quantitativ oder auch qualitativ zu fassen. Andererseits erschwert bzw. verunmöglicht die derzeitige Situation den Nutzerinnen und Nutzern, "gute", d. h. seriöse und verlässliche Angebote zu identifizieren.

Aufgrund fehlender einheitlicher Definitionen und verbindlicher Qualitätsstandards finden sich zahlreiche Gesundheits-Apps, die kurzfristige gesundheitsbezogene Erfolge versprechen, wissenschaftlich nicht (hinreichend) fundiert oder hinsichtlich des Datenschutzes bedenklich sind. Gleichzeitig fehlt es an aktuellen Untersuchungen mit zielgruppenspezifischen Befunden zum Stellenwert und zur Nutzung von GesundheitsApps, die über reine Anwendungsstudien hinausweisen. Vorliegende Studien zeigen, dass eine Smartphoneaffinität und das Interesse an (Gesundheits-)Apps nicht automatisch bedeuten, dass die digitalen
Angebote nach dem Download auch genutzt werden.

Angesichts der dünnen Forschungslage kann davon ausgegangen werden, dass die Potenziale von Gesundheits-Apps sowohl von den Anbietern als auch von den Nutzerinnen und Nutzern noch nicht voll ausgeschöpft werden. In der Herstellung von Transparenz (u.a. in Bezug auf Datenerfassung, -verarbeitung, -schutz und die wissenschaftliche Fundierung), der (Weiter-)Entwicklung von Qualitätsstandards sowie in der Förderung einer digitalen Gesundheitskompetenz, einschließlich der Bereitstellung zielgruppenadäquater Tools für eine Einschätzung und Auswahl von Gesundheits-Apps, werden zentrale Ansatzpunkte gesehen, um die Potenziale von Gesundheits-Apps insbesondere mit Blick auf Kinder und Jugendliche besser nutzbar zu machen.

\section{Schlüsselwörter}

Präventions-Apps · Kinder und Jugendliche . Angebot und Nutzung · Digitale Gesundheitskompetenz - Digitale Gesundheitsangebote

\section{Untapped potential—health apps for children and adolescents}

\section{Abstract}

Health apps are increasingly getting attention and gaining support from policymakers. The market is currently characterized by a very high level of vibrancy and confusion. On the one hand, it is impossible to define the range of health apps quantitatively or qualitatively. On the other hand, it is difficult or impossible for users to identify "good" health apps in the sense of serious and reliable products and services.

Due to the lack of common definitions and obligatory quality standards, numerous health apps promise short-term healthrelated success, are not scientifically (sufficiently) sound, or are questionable concerning data protection. At the same time, there is a lack of current studies with targetgroup-specific findings on the significance and use of health apps that go beyond pure application studies. Existing studies show that an affinity for smartphones and interest in (health) apps does not automatically mean that digital services are also used after downloading.

Given the poor research available, it can be assumed that both providers and users will not fully exploit the potential of health apps. The creation of transparency (e.g., about data collection, processing, protection, and scientific foundation), (further) development of quality standards, as well as the promotion of digital health literacy, including the provision of tools appropriate to target groups for the assessment and selection of health apps, are seen as central starting points for making the potential of health apps more usable, especially with regard to children and adolescents.

\section{Keywords}

Prevention apps - Children and adolescents . Offer and use - Digital health literacy · Digital health programmes 
de nach der aktuellen Nutzung gefragt, verringerte sich der Anteil an Gesundheits-App-Nutzerinnen und -Nutzern allerdings auf $25 \%$. Im Durchschnitt nutzten die Befragten 1,6 GesundheitsApps auf ihrem Smartphone, am häufigsten zu den Themen Fitness, Ernährung, Schlaf und Menstruation. 52\% haben Gesundheits-Apps zu einem bis drei verschiedenen Gesundheitsthemen genutzt. Gut ein Drittel der Befragten (34\%) hatte keine Erfahrungen mit GesundheitsApps. Diejenigen, die Gesundheits-Apps nutzen oder genutzt haben, bewerteten diese überwiegend $(76 \%)$ als hilfreich (27\% sogar als sehr hilfreich). Altersund geschlechterbezogene Unterschiede zeigten sich dahin gehend, dass die Gesundheits-Apps überwiegend von den 18- bis 22 -Jährigen ( $76 \%$ vs. $49 \%$ der 14 bis 17-Jährigen) und von den weiblichen Befragten ( $71 \%$ vs. $57 \%$ der männlichen Befragten) genutzt wurden.

Eine französische Studie unter 18bis 24-jährigen Studierenden stellte fest, dass $35 \%$ der Befragten mindestens eine Gesundheits-App auf ihrem Smartphone haben [19]. $63 \%$ nutzten diese gelegentlich, $28 \%$ oft und $10 \%$ nie. Inhaltlich handelte es sich vor allem um Bewegungs-Apps (49\%) oder Apps, die das allgemeine Gesundheitsverhalten monitoren und unterstützen (41\%). Seltener wurden Schlaf-Apps (17\%), Ernährungs-Apps (8\%), Wellness-Apps (z. B. Yoga, 6\%) oder Apps zum Thema Frauengesundheit (4\%) genutzt. Vereinzelt nutzten die Studierenden Apps zum Thema Sucht oder Allergie. Mehr als ein Drittel der Befragten hatte noch keine Gesundheits-App heruntergeladen, verwies jedoch auf die Möglichkeit zur Nutzung vorinstallierter Apps, wie z.B. die Health-App auf dem iPhone. Zudem zeigte sich, dass weibliche Studierende fast doppelt so häufig gesundheitsbezogene Apps nutzen wie ihre männlichen Kommilitonen. Mögliche Gründe für die insgesamt vergleichsweise geringe Nutzung von Gesundheits-Apps in der Untersuchungsgruppe werden in dem Aufwand für die Dateneingabe sowie in den geringen Speicher- und Batteriekapazitäten der Smartphones gesehen.

Verschiedene qualitative Studien geben Hinweise auf die zweckgebundene
Nutzung sowie auf mögliche Gründe, die in einer Nichtnutzung von Gesundheits-Apps resultieren können. Eine qualitative Studie unter 16- bis 25-Jährigen in Australien $(n=30)$ zeigt beispielsweise, dass digitale Gesundheitsangebote für unterschiedliche Zwecke genutzt werden [15]. So sind Suchmaschinen, Gesundheitswebseiten und auch Social-MediaAngebote wie YouTube oder soziale Netzwerkseiten wichtige Informationsquellen neben Ärzten, Familie und Freunden, während Wearables und Apps zum Tracken gesundheitsbezogenen Verhaltens (Schlafen, Bewegung etc.) und zur Förderung des eigenen Wohlbefindens genutzt werden. In einer weiteren qualitativen Studie unter Jugendlichen im Alter von 13 bis 18 Jahren $(n=20)$ finden sich zudem Hinweise, dass Jugendliche z.T. noch nie etwas von Gesundheits-Apps gehört haben, zudem nicht wussten, dass auf ihrem Smartphone gesundheitsbezogene Funktionen angeboten werden, sich auch nicht für derartige Angebote interessieren, geschweige denn danach suchen oder derartige Angebote als langweilige, z.T. uncoole Angebote betrachten, die an Erwachsene gerichtet sind [5]. In ähnliche Richtung weisen die Antworten von Jugendlichen in einer explorativen Onlinebefragung, aus denen hervorging, dass manche Heranwachsende entweder keinen Bedarfhaben („Bin 11 Jahre, brauche so etwas nicht"), Gesundheits-Apps $\mathrm{zu}$ nutzen, oder auch z.T. keine geeigneten oder für sie passenden Angebote (er)kennen (,Weil ich keine guten Apps kenne“; „Weil ich keine Passende finde“; „Ich weiß nicht, welche passend ist") oder mitunter nicht über die erforderlichen technischen Voraussetzungen, d.h. vor allem genügend Speicherplatz, verfügen [2].

Insgesamt deuten die Ergebnisse der vorliegenden Studien daraufhin, dass Gesundheits- und insbesondere Präventions-Apps im Medien- und App-Repertoire von Heranwachsenden offensichtlich (noch) keine besonders große Rolle spielen. Dies kann zum einen darauf zurückzuführen sein, dass viele Kinder und Jugendliche keine Notwendigkeit sehen oder kein Interesse haben, sich im Kontext ihrer Smartphonenutzung (präventiv) mit gesundheitsbezogenen Themen auseinanderzusetzen. Zum anderen ist $\mathrm{zu}$ berücksichtigen, dass den Heranwachsenden ein sehr umfangreiches Angebot an kostenlosen Apps zur Verfügung steht, das ihren Bedürfnissen nach Kommunikation und Unterhaltung offensichtlich eher entspricht [5].

\section{Potenziale, Grenzen und Risiken von Gesundheits-Apps für Kinder und Jugendliche}

Apps bieten zweifellos vielfältige Möglichkeiten für die Gesundheitskommunikation und -förderung (s. - Tab. 1). Die technischen Möglichkeiten erlauben es, Inhalte multimedial, interaktiv, personalisiert und adaptiv zu präsentieren. Die Möglichkeiten werden jedoch von den Anbietern in sehr unterschiedlicher Weise genutzt, z. T. auch gar nicht ausgeschöpft, sodass das Angebot an Gesundheits-Apps allein hinsichtlich der technischen Umsetzung gravierende Unterschiede aufweist. Hinzu kommen Design, Konzeption und Funktionalitäten, die mit darüber entscheiden, ob eine App einmalig oder längerfristig genutzt wird und ihr gesundheitsförderndes Potenzial (oder ihr Risiko) entsprechend entfalten kann.

\section{Potenziale}

Vorteile von Gesundheits- bzw. Präventions-Apps werden u. a. in der zeit- und ortsungebundenen Verfügbarkeit, dem zielgruppenspezifischen Zugang zu jüngeren (aber auch zu schwer erreichbaren) Gruppen, der unmittelbaren unterstützenden Funktion, der Anonymität, dem individuell zugeschnittenen Inhalt, in den geringeren Kosten sowie wachsenden Serviceangeboten sowie in der Effizienz gesehen [7, 8]. Für Heranwachsende bieten digitale Angebote zudem die Möglichkeit, sich mit gesundheitsbezogenen Entwicklungsthemen und Fragen (z.B. zu körperlichen Veränderungen, Sexualität, Ernährung, psychischer Gesundheit) auseinanderzusetzen [20].

Verschiedene Studien attestieren digitalen Anwendungen ein gesundheitsförderndes Potenzial, sowohl in Bezug auf das behandelte Thema als auch im Hinblick auf den emotionalen Status, 
Tab. 1 Potenziale und Grenzen von Präventions-Apps für Kinder und Jugendliche

Präventions-Apps

\begin{tabular}{|c|c|}
\hline Zielgruppe & Smartphoneaffine, gesundheitsinteressierte Zielgruppen \\
\hline Themen & Thematisch sind keine Grenzen gesetzt \\
\hline \multirow{3}{*}{$\begin{array}{l}\text { (Gesundheits- } \\
\text { bezogene) Ziele }\end{array}$} & Unterstützung gesunder Verhaltensweisen \\
\hline & Sensibilisierung/Awareness für gesundheitsbezogene Themen \\
\hline & Informationsvermittlung \\
\hline \multirow[t]{5}{*}{ Potenziale } & Zeit- und ortsungebundene Verfügbarkeit \\
\hline & Anregung zur Auseinandersetzung mit gesundheitsbezogenen Themen \\
\hline & Personalisierbar und adaptiv \\
\hline & Geringe Kosten \\
\hline & Anonymität \\
\hline \multirow[t]{4}{*}{ Grenzen } & Zugang ist vergleichsweise voraussetzungsvoll \\
\hline & $\begin{array}{l}\text { Angebote müssen gesucht, gefunden, ausgewählt, heruntergeladen und } \\
\text { schließlich genutzt werden }\end{array}$ \\
\hline & Großes konkurrierendes Angebot an kostenlosen Unterhaltungs-Apps \\
\hline & $\begin{array}{l}\text { Angebot an seriösen Präventions-Apps ist unübersichtlich und intranspa- } \\
\text { rent }\end{array}$ \\
\hline \multirow[t]{3}{*}{ Risiken } & Verbreitung ungesicherter, mitunter falscher Informationen \\
\hline & Datenschutz und -sicherheit \\
\hline & $\begin{array}{l}\text { Unerwünschte Nebenwirkungen (z. B. Fixierung auf die Erfassung von } \\
\text { Messwerten, Erhöhung der Smartphonenutzungsdauer) }\end{array}$ \\
\hline
\end{tabular}

die Zufriedenheit und das persönliche Selbstwertgefühl [21]. Besonders betont werden die motivierenden Möglichkeiten solcher Angebote, die ein gewisses $\mathrm{Maß}$ an Gamification [22] aufweisen (siehe hierzu auch den Beitrag von Tolks et al. in diesem Themenheft). In einigen Studien wird der gesundheitsfördernde Mehrwert von unterhaltsamen bzw. spielerischen Anwendungen allerdings infrage gestellt, da der Gesundheitsbezug nicht immer erkennbar oder der Anteil spielerischer Elemente (Grad der Gamification) mitunter zu hoch ist oder weil keine Gesundheitsexpertinnen und -experten einbezogen wurden [21].

\section{Grenzen}

Auch wenn die Möglichkeiten von Apps für die Prävention und Gesundheitsförderung auf den ersten Blick attraktiv erscheinen, so sind ihnen auch Grenzen gesetzt [21], z. B. dadurch, dass die von den Kinder und Jugendlichen (oder auch von Eltern) überhaupt erst gesucht, gefunden, ausgewählt und schließlich heruntergeladen werden müssen [3, 9]. Die gesundheitsbezogenen Angebote stehen dabei in großer Konkurrenz zu einer Vielzahl an zumeist kostenlosen Angeboten, die oftmals stärker die (unterhaltungsbezogenen) Bedürfnisse und Interessen der Zielgruppe ansprechen und aus ihrer Sicht weniger pädagogisch anmuten [3, 9].

Neben der Auffindbarkeit und der bewusst zu treffenden Auswahlentscheidung für eine App sind der „Erfolg“ und eine längerfristige Wirkung einer AppAnwendung auch von der Nutzungshäufigkeit und -dauer abhängig. Bislang ist allerdings noch unklar, wie lange das Interesse bei den Nutzerinnen und Nutzern und insbesondere bei Kindern und Jugendlichen an einem Angebot vorhält $[1,21]$. Von einer höheren Motivation aufseiten der Nutzerinnen und Nutzer und einer entsprechend längeren Nutzungsdauer kann vor allem bei solchen Angeboten ausgegangen werden, die der Therapieunterstützung dienen (Medizin-Apps) oder zum Selbstmanagement genutzt werden können.

\section{Risiken}

Auch wenn die App-Technologien viele faszinierende Möglichkeiten bieten, sollten die möglichen Risiken nicht außer Acht gelassen werden (- Tab. 1). Neben den gesundheitsbezogenen Risiken durch z. B. fachlich nicht fundierte, mitunter falsche Informationen oder auch durch Fehlinterpretationen von Informationen, Messwerten oder datenbasierten Empfehlungen [2] sind die Nutzerinnen und Nutzer mit Risiken konfrontiert, die sich u. a. durch die Preisgabe, Aggregierung und (kommerzielle) Auswertung gesundheitsbezogener Daten für die informationelle Selbstbestimmung ergeben [23]. Das Angebot an GesundheitsApps ist bezüglich der Datenschutzpraktiken ebenfalls uneinheitlich und unübersichtlich. In den seltensten Fällen wird den Nutzerinnen und Nutzern in verständlicher und nachvollziehbarer Weise nahegebracht, welche Daten $\mathrm{zu}$ welchen Zwecken erhoben werden, in welcher Form sie ihre Einwilligung geben und wie bereits erhobene Daten wieder gelöscht werden können. Insbesondere mit Blick auf vulnerable Zielgruppen wie Kinder und Jugendliche ergibt sich diesbezüglich ein besonderer Schutzbedarf.

Weitere mögliche Risiken können unerwünschte (Neben-)Wirkungen (z.B. Fixierung auf die Erfassung von Gesundheitsdaten und Zunahme der Smartphonenutzungsdauer) oder ausbleibende erwünschte Wirkungen sein [1]. Studien $\mathrm{zu}$ intendierten Effekten als auch $\mathrm{zu}$ den Risiken und (unerwünschten) Nebenwirkungen sowie wissenschaftliche Begleitstudien zu Gesundheits-Apps sind noch vergleichsweise rar $[1,7]$.

\section{Fazit und Handlungs- empfehlungen}

Die Digitalisierung geht auch in der Gesundheitskommunikation mit gravierenden Veränderungen einher. Rideout und Fox sprechen von einer „digital health revolution", die für die gegenwärtige $\mathrm{Ge}$ neration folgenreich sei, da sie die Art und Weise, wie (gesundheitsbezogene) Informationen gesucht, Geschichten und Erfahrungen geteilt und über das Thema Gesundheit miteinander gesprochen wird, grundlegend verändere [18, S. 78]. Wartella et al. sehen in dieser Entwicklung die Chance, dass sich Jugendliche intensiver mit ihrer Gesundheit auseinandersetzen und vor dem Hintergrund der verfügbaren Daten und Informationen 
selbstbestimmt gesündere Entscheidungen treffen (können). Wie sich diese digitale Veränderung auf das Gesundheitsverhalten auswirken wird, wird allerdings nur in langfristigen Untersuchungen $\mathrm{zu}$ ermitteln sein [16]. Montagni et al. verweisen in diesem Zusammenhang auch auf die Notwendigkeit von Studien zur Verbreitung, (Nicht-)Nutzung und Akzeptanz von Gesundheits-Apps, die auch die Faktoren berücksichtigen, die Aufschluss darüber geben, warum eine App mitunter nicht weiter genutzt wird [19].

\section{Ungenutzte Potenziale}

Insgesamt scheinen die Potenziale von Gesundheits-Apps mit Blick auf Kinder und Jugendliche noch nicht ausgeschöpft - weder von den Anbietern noch von den Nutzerinnen und Nutzern. Aktuell ist die Situation von einer hohen Aufbruchsstimmung geprägt, in der vieles ausprobiert, getestet und mitunter wieder verworfen wird. Entsprechend ist das Angebot an Gesundheitsinformationen und -Apps durch eine sehr große Unübersichtlichkeit geprägt. Hinzu kommt, dass bei den meisten Nutzerinnen und Nutzern nur wenig Wissen darüber vorhanden ist, wie Gesundheits-Apps in die Stores gelangen und nach welchen Algorithmen diese angezeigt werden. Ähnlich wie bei der Google-Suche wirken sich beispielsweise auch im Google-PlayStore vorherige Suchanfragen oder AppDownloads auf die Anzeige von Suchergebnissen aus.

\section{Transparenz und Orientierungs- hilfen}

Ein erster wichtiger Schritt wäre daher, die Transparenz im Bereich der Gesundheits-Apps zu erhöhen. Dies könnte über entsprechende Informationen in den einzelnen Stores stattfinden. Orientierung bieten könnten z.B. auch App-Datenbanken (etwa ähnlich der Datenbank „Apps für Kinder" des Deutschen Jugendinstitutes) oder App-Bewertungen, wie z. B. auf handysektor.de (angepasst für oder erweitert um Gesundheits-Apps), Qualitätssiegel oder „App-Beipackzettel“ [2]. Auch wenn bereits einige Überlegungen und Bewertungshilfen, wie z.B.
AppCheck für Diabetes- und PneumoApps (Zentrum für Telematik und Telemedizin $\mathrm{GmbH}$, www.appcheck.de) oder der HealthOn-App-Ehrenkodex (Initiative Präventionspartner, https:// www.healthon.de/ehrenkodex), vorliegen, steht die Entwicklung umfassender und valider Instrumente zur Qualitätsbewertung noch aus [1]. Zudem stoßen derartige angebotsbezogene Orientierungshilfen angesichts der hohen Dynamik des Marktes sehr schnell an ihre Grenzen. Sinnvoll und nachhaltiger scheint daher, den Nutzerinnen und Nutzern (im vorliegenden Fall Eltern, Kindern und Jugendlichen) Informationen und Kriterien an die Hand zu geben, wie sie selbst gute (im Sinne von seriös und verlässlich) Angebote identifizieren können, welchen gesundheitlichen Mehrwert die Nutzung einer App für sie haben kann und was sie bei der Nutzung von GesundheitsApps (z.B. hinsichtlich der Preisgabe gesundheitsbezogener Daten) beachten sollten. Einige Orientierungsangebote und Entscheidungshilfen in Form von Checklisten liegen bereits vor, wie z.B. die „Checkliste für die Nutzung von Gesundheits-Apps" vom Aktionsbündnis Patientensicherheit [24] oder die von dem Universitätsklinikum Freiburg entwickelte Checkliste „Gesundheits-Apps: Bewusst auswählen ist das A und $\mathrm{O}^{\text {“ }}$ [25]. Zudem wurden in jüngster Zeit umfassende Kriterienkataloge erstellt, die eine Bewertung von Apps erleichtern sollen (z.B. für Medizin-Apps, APPQ, [26]). Allerdings sind viele der z.T. sehr umfassenden Angebote nicht für jüngere Zielgruppen (oder Eltern) geeignet. Hier wären zielgruppenadäquate Orientierungs- und Unterstützungsangebote sinnvoll und wünschenswert, die einerseits den Mediennutzungspraktiken der Heranwachsenden Rechnung tragen und andererseits die Jugendlichen über die Potenziale und Herausforderungen von Gesundheits-Apps informieren sowie zu einer bewussteren Auswahl und Nutzung motivieren [2].

\section{Förderung einer digitalen Gesundheitskompetenz}

Die Sensibilisierung für einen bewussteren Umgang mit digitalen Gesundheitsangeboten verweist schließlich auf die Notwendigkeit der Förderung der allgemeinen digitalen Gesundheitskompetenz („digital health literacy“), verstanden als "the ability to seek, find, understand and appraise health information from electronic sources and apply the knowledge gained to addressing or solving a health problem" $[27$, S. 3]. Angesichts der Ausweitung von Trends, wie z.B. Self-Tracking und Selbstoptimierung, sowie erhöhten Anforderungen an das digitale Selbstmanagement ergeben sich neue Anforderungen an eine digitale Gesundheitskompetenz u.a. im Hinblick auf Fragen der (bewussten und unbewussten) Datenerhebung, -verarbeitung und -verwertung. Erst Ende Oktober 2019 kündigte Facebook an, künftig Funktionen anzubieten, die an Vorsorgeuntersuchungen erinnern [28], und Anfang November wurde öffentlich, dass Google für zwei Milliarden Dollar Fitbit gekauft hat [29]. Angesichts dieser und ähnlicher Pläne datenstarker Unternehmen sowie der verbesserten Möglichkeiten zur anwendungsübergreifenden Auswertung großer Datenmengen (Stichwort: Big Data) werden Themen wie Datenschutz und Datensicherheit, aber auch langfristige Folgen für die Gesundheitsversorgung und für die informationelle Selbstbestimmung noch dringender $\mathrm{zu}$ diskutieren sein.

\section{Korrespondenzadresse}

\section{Dr. Claudia Lampert}

Leibniz-Institut für Medienforschung, HansBredow-Institut (HBI)

Rothenbaumchaussee 36, 20148 Hamburg, Deutschland

c.lampert@leibniz-hbi.de

Funding. Open Access funding provided by Projekt DEAL. 


\section{Einhaltung ethischer Richtlinien}

Interessenkonflikt. C. Lampert gibt an, dass kein Interessenkonflikt besteht.

Für diesen Beitrag wurden vom Autor keine Studien an Menschen oder Tieren durchgeführt. Für die aufgeführten Studien gelten die jeweils dort angegebenen ethischen Richtlinien.

Open Access Dieser Artikel wird unter der Creative Commons Namensnennung 4.0 International Lizenz veröffentlicht, welche die Nutzung, Vervielfältigung, Bearbeitung, Verbreitung und Wiedergabe in jeglichem Medium und Format erlaubt, sofern Sie den/die ursprünglichen Autor(en) und die Quelle ordnungsgemäß nennen, einen Link zur Creative Commons Lizenz beifügen und angeben, ob Änderungen vorgenommen wurden.

Die in diesem Artikel enthaltenen Bilder und sonstiges Drittmaterial unterliegen ebenfalls der genannten Creative Commons Lizenz, sofern sich aus der Abbildungslegende nichts anderes ergibt. Sofern das betreffende Material nicht unter der genannten Creative Commons Lizenz steht und die betreffende Handlung nicht nach gesetzlichen Vorschriften erlaubt ist, ist für die oben aufgeführten Weiterverwendungen des Materials die Einwilligung des jeweiligen Rechteinhabers einzuholen.

Weitere Details zur Lizenz entnehmen Sie bitte de Lizenzinformation auf http://creativecommons.org/ licenses/by/4.0/deed.de.

\section{Literatur}

1. Albrecht UV (2016) Kurzfassung. In: Medizinische Hochschule Hannover (Hrsg) Chancen und Risiken von Gesundheits-Apps (CHARISHMA), S 14-47

2. Lampert C, Scherenberg V (2019) HealthApps4Teens Report. Hamburg, Bremen

3. Lampert C, Voß M (2018) Möglichkeiten und Grenzen digitaler Gesundheitsangebote im Unterhaltungsformat. In: Scherenberg V, Pundt J (Hrsg) Digitale Gesundheitskommunikation Zwischen Meinungsbildung und Manipulation. APOLLON University Press, Bremen

4. Scherenberg V, Kramer U (2013) Digitale Prävention - Über Chancen und Risiken von GesundheitsApps. Mabuse 205:43-45

5. Chan A, Kow R, Cheng JK (2017) Adolescents' perceptions on smartphone applications (apps) for health management. J Mob Technol Med 6:47-55. https://doi.org/10.7309/jmtm.6.2.6

6. Scherenberg V, Liegmann K (2018) Gesundheits-Apps: Möglichkeiten und Grenzen der Gesundheitskommunikation. In: Digitale Gesundheitskommunikation. Zwischen Meinungsbildung und Manipulation. APOLLON University Press, Bremen, S245-273

7. Grist R, Porter J, Stallard P (2017) Mental health mobile apps for preadolescents and adolescents: a systematic review. J Med Internet Res 19:e176. https://doi.org/10.2196/jmir.7332

8. Olff M (2015) Mobile mental health: a challenging research agenda. Eur J Psychotraumatol 6:27882. https://doi.org/10.3402/ejpt.v6.27882

9. Lampert C, Voß M (2018) Gesundheitsbezogene Apps für Kinder - Ergebnisse des Projekts HealthApps4Kids. Hans-Bredow-Institut, Hamburg
10. Murray A, Lyle J (2015) Patient adoption of mHealth. Use, evidence and remaining barriers to mainstream acceptance. IMS Institute for Healthcare Informatics, Parsippany, NJ

11. Initiative Präventionspartner, Kramer U (2017) Das „Gesundheits-App-Dilemma“. https://www. healthon.de/infografiken/2017/01/das- $\% E 2 \% 80$ $\% 9 E$ Eesundheits-app-dilemma\%E2\%80\%9C. Zugegriffen: 15. Apr. 2018

12. BITKOM - Bundesverband Informationswirtschaft (2019) Kinder und Jugendliche in der digitalen Welt

13. Hasebrink U, Lampert C, Thiel K (2019) OnlineErfahrungen von 9-bis 17-Jährigen. Ergebnisse der EU Kids Online-Befragung in Deutschland. HansBredow-Institut, Hamburg

14. Medienpädagogischer Forschungsverbund Südwest (2018) JIM-Studie 2018: Jugend, Information, Medien: Basisuntersuchung zum Medienumgang 12- bis 19-Jähriger. Medienpädagogischer Forschungsverbund Südwest, Stuttgart

15. Lupton D (2018) 'Better understanding about what's going on': young Australians' use of digital technologies forhealth and fitness. SportEduc Soc https://doi.org/10.1080/13573322.2018.1555661

16. Wartella E, Rideout V, Montague H, BeaudoinRyan L, Lauricella A (2016) Teens, health and technology: a national survey. Media Commun 4:13. https://doi.org/10.17645/mac.v4i3.515

17. Wartella $E$, Rideout V, Zupancic $H$, BeaudoinRyan L, Lauricella A (2015) Teens, health, and technology. Report of the Center on Media and Human Development, School of Communication. Northwestern University, Evanston

18. Rideout V, Fox S, Well Being Trust (2018) Digital health practices, social media use, and mental wellbeing among teens and young adults in the U.S. Providence St. Joseph Health Digital Commons

19. Montagni I, Cariou T, Feuillet T, Langlois E, Tzourio C (2018) Exploring digital health use and opinions of university students: field survey study. JMIR Mhealth Uhealth 6:e65. https://doi.org/10.2196/ mhealth.9131

20. Lampert C (2018) Gesundheitsangebote für Kinder und Jugendliche im App-Format. Präv Gesundheitsf 13:280-284. https://doi.org/10. 1007/s11553-018-0665-y

21. Sardi L, Idri A, Fernández-Alemán JL (2017) A systematic review of gamification in e-Health. J Biomed Inform 71:31-48. https://doi.org/10. 1016/j.jbi.2017.05.011

22. Deterding S, Khaled R, Nacke L, Dixon D (2011) Gamification: toward a definition. In: CHI 2011 Gamification Workshop Proceedings, S 12-15

23. Die Bundesbeauftragte für den Datenschutz und die Informationsfreiheit (2018) Gesundheits-Apps. BfDI, Bonn

24. APS e.V. (2018) Digitalisierung und Patientensicherheit. Checkliste für die Nutzung von Gesundheitsapps. APS e.V., Berlin

25. Techniker Krankenkasse (2018) GesundheitsApps: Bewusst auswählen ist das $\mathrm{A}$ und $\mathrm{O}$. https://www.tk.de/techniker/gesundheit-undmedizin/kompetent-als-patient/gesundheitsapps-bewusstes-auswaehlen-2010050. Zugegriffen: 17. Nov. 2019

26. Bertelsmann Stiftung (2019) AppQ. GütekriterienKernset für mehr Qualitätstransparenz bei digitalen Gesundheitsanwendungen. Bertelsmann Stiftung, Gütersloh

27. European Commission (2014) Flash Eurobarometer 404 "European citizens" digital health literacy. European Union, n.p.
28. Jee C (2019) Facebook will now remind you to get health checkups (if you want): https://www.technologyreview.com/2019/10/ 29/102487/facebook-will-now-remind-you-toget-health-check-ups-if-you-want/. Zugeriffen: 3. Nov. 2019

29. kein Autor (2019) Google will fitter werden. In: Zeit Online-Digit. https://www.zeit.de/digital/mobil/ 2019-11/fitbit-smartwatch-anbieter-googleuebernahme.Zugegriffen:3.Nov. 2019 